\title{
THE LEAST COMMUTATIVE CONGRUENCE ON A SIMPLE REGULAR $\omega$-SEMIGROUP $\dagger$
}

\author{
by C. BONZINI, A. CHERUBINI and B. PIOCHI
}

(Received 22 March, 1988; revised 17 October, 1988)

Introduction. Piochi in [10] gives a description of the least commutative congruence $\lambda$ of an inverse semigroup in terms of congruence pairs and generalizes to inverse semigroups the notion of solvability. The object of this paper is to give an explicit construction of $\lambda$ for simple regular $\omega$-semigroups exploiting the work of Baird on congruences on such semigroups. Moreover the connection between the solvability classes of simple regular $\omega$-semigroups and those of their subgroups is studied.

As usual $\sigma$ indicates the least group congruence. $\mathbb{H}$ and $\mathbb{D}$ the Green's relations, $\mathbb{N}$ the set of non negative integers, $\mathbb{Z}$ the additive group of the integers. For notations and definitions not given in this paper the reader is referred to [9].

\section{Preliminary results.}

Definition 1. An $\omega$-semigroup $S$ is a semigroup whose set $E$ of idempotents form an $\omega$-chain

$$
e_{0}>e_{1}>\ldots>e_{n}>\ldots
$$

under the natural order defined on $E$ by the rule $e \geq f$ if and only if $e f=f=f e$.

For a regular $\omega$-semigroup. Munn in [6] proved the following result.

THEOREM A. Let $S$ be a regular $\omega$-semigroup.

If $S$ has no kernel, then it is the union of an $\omega$-chain of groups.

If the kernel of $S$ coincides with $S$, then $S$ is a simple regular $\omega$-semigroup.

If $S$ has a proper kernel, then $S$ is a (retract) ideal extension of a simple regular $\omega$-semigroup $K$ by a finite chain of groups with 0 adjoined $H^{0}$. Moreover this extension is determined by means of a homomorphism of $H$ into the group of units of $K$.

Piochi in [10] characterized (by means of congruence pairs) the least commutative congruence of an inverse semigroup, proving

Theorem B ([10], Th. 2.4 and Th. 2.6). Let $S$ be an inverse semigroup and $E$ its semilattice of idempotents. Define on $E$ the relation $e \sim f$ if and only if there exist $a, b \in S$ such that $e=a b b^{-1} a^{-1}, f=b a a^{-1} b^{-1}$ and denote by $\lambda_{E}$ the transitive closure of $\sim$. Denote by $S^{\prime}$ the subsemigroup of $S$ generated by the elements $[a, b]=a b a^{-1} b^{-1}$ with $a, b \in S$ and put

$$
\partial(S)=\left\{a \in S \mid a^{-1} a \lambda_{E} \text { e for some } e \in E \text { and } a e \in S^{\prime}\right\} .
$$

Then $\left(\lambda_{E}, \partial(S)\right)$ is a congruence pair and the congruence associated with it is the least commutative congruence on $S$.

Henceforward the least commutative congruence on a semigroup $S$ will be denoted by $\lambda_{s}$ (or simply by $\lambda$ ). We remark that the congruence $\lambda$ is denoted $\gamma$ in [10]; here we changed notation, in order to avoid confusion with the mappings $\gamma_{i}$ of Theorem C below.

† Work supported by M.P.I.

Glasgow Math. J. 32 (1990) 13-23. 
The main aim of this paper is to give an explicit construction of $\lambda$ for a simple regular $\omega$-semigroup. The construction of $\lambda$ for the non-simple case is a result of a routine nature but adds to the technical problems, hence here is deleted; however it can be found in [4].

2. The least commutative congruence on a simple regular $\omega$-semigroup. Several authors, e.g. Kocin [5] and Munn [6], gave structure theorems for simple regular $\omega$-semigroups. The one given by Munn is the following

Theorem C. Let $d$ be a positive integer and let $\left\{G_{i} \mid i=0, \ldots, d-1\right\}$ be a set of $d$ pairwise disjoint groups. Let $\gamma_{d-1}$ be a homomorphism of $G_{d-1}$ into $G_{0}$ and, if $d>1$, let $\gamma_{i}$ be a homomorphism of $G_{i}$ into $G_{i+1}(i=0, \ldots, d-2)$. For every $n \in \mathbb{N}$ let $\bar{n}$ denote the integer equivalent to $n$ modulo $d$, belonging to $\mathbb{N}$ and less than $d$ and let $\gamma_{n}=\gamma_{\bar{n}}$. For $m, n \in \mathbb{N}$ and $m<n$ write

$$
\alpha_{m, n}=\gamma_{m} \gamma_{m+1} \ldots \gamma_{n-1}
$$

and for all $n \in \mathbb{N}$ let $\alpha_{n, n}$ denote the identity automorphism of $G_{\bar{n}}$. Let $S$ be the set of the ordered triples $\left(m, a_{i}, n\right)$, where $m, n \in \mathbb{N}, 0 \leq i \leq d-1$ and $a_{i} \in G_{i}$. Define a multiplication in $S$ by the rule that

$$
\left(m, a_{i}, n\right)\left(p, b_{j}, q\right)=\left(m+p-r,\left(a_{i} \alpha_{u, w}\right)\left(b_{j} \alpha_{v, w}\right), n+q-r\right)
$$

where $r=\min \{n, p\}, u=n d+i, v=p d+j$ and $w=\max \{u, v\}$. Denote the so formed groupoid by $S\left(d, G_{i}, \gamma_{i}\right)$. Then $S\left(d, G_{i}, \gamma_{i}\right)$ is a simple regular $\omega$-semigroup with exactly $d \mathbb{D}$-classes and any simple regular $\omega$-semigroup is isomorphic to a semigroup $S\left(d, G_{i}, \gamma_{i}\right)$. For $n \in \mathbb{N}$ and $i=0, \ldots, d-1$ write $e_{i}^{n}=\left(n, e_{i}, n\right)$, where $e_{i}$ is the identity of the group $G_{i}$. The elements $e_{i}^{n}$ are the idempotents of $S\left(d, G_{i}, \gamma_{i}\right)$ and we have

$$
e_{0}^{0}>e_{1}^{0}>\ldots>e_{d-1}^{0}>e_{0}^{1}>\ldots>e_{d-1}^{1}>e_{0}^{2}>\ldots
$$

Notation. In the remainder of the paper $\bar{n}$ will denote, as in previous theorem, the integer equivalent to $n$ modulo $d$, belonging to $\mathbb{N}$ and less than $d$, and, for every $i \in \mathbb{N}$, the endomorphism $\alpha_{i, i+d}$ of $G_{i}$ will be indicated by $\alpha_{i}$.

REMARK 2.1. For every $i, j \in \mathbb{N}$ with $i<j$ we have obviously $\alpha_{i}=\alpha_{\bar{i}}$ and, putting $i=m d+\bar{i}, j=n d+\bar{j}, \alpha_{i, j}=\alpha_{\bar{i}, j} \alpha_{j}^{n-m}$ if $\bar{i} \leq \bar{j}$ and $\alpha_{i, j}=\alpha_{\bar{i}, \bar{j}+d} \alpha_{\bar{j}}^{n-m-1}$ if $\bar{i}>\bar{j}$.

Remark 2.2. For fixed $i$ satisfying $0 \leq i \leq d-1$, put

$$
S_{i}=\left\{\left(m, a_{i}, n\right) \mid m, n \in \mathbb{N}, a_{i} \in G_{i}\right\} .
$$

Then $S_{i}$ is a bisimple inverse semigroup of $S([2]$, p. 462) and the Reilly multiplication ([7], formula (1)) applies with $\alpha=\alpha_{i}$.

LemMa 2.3. Let $S=S\left(d, G_{i}, \gamma_{i}\right)$ be a simple regular $\omega$-semigroup. Then its least commutative congruence $\lambda$ is a group congruence contained in $\sigma \vee \mathbb{H}$.

Proof. First we recall that the congruence $\lambda_{E}$ defined in Th. B is, by Th. 2.2 of [3], a uniform congruence of $E$. Moreover, for every $m, n \in \mathbb{N}$ and for every $i$ such that $0 \leq i \leq d-1$, we have $e_{i}^{m} \lambda_{E} e_{i}^{n}$. In fact, putting $a=\left(m, e_{i}, n\right), b=\left(n, e_{i}, m\right)$ we have $e_{i}^{m}=a b b^{-1} a^{-1}$ and $e_{i}^{n}=b a a^{-1} b^{-1}$. Hence, by the remarks preceding Lemma 2.1 of [3], we immediately deduce that $\lambda_{E}$ is the universal congruence $\omega_{E}$ on $E$, hence $\lambda$ is a group 
congruence. Finally we remark that, since $S / \sigma \vee \mathbb{H} \cong \mathbb{Z}$ (see [2], Corollary 3.1), $\sigma \vee \mathbb{H}$ is a commutative congruence; so $\lambda$ is contained in $\sigma \vee A$.

Corollary 2.4. If $\rho$ is a commutative congruence on $S=S\left(d, G_{i}, \gamma_{i}\right)$, then $\rho$ is a group congruence.

Definition $2.5 \quad([2], \quad$ p. 463$)$. Let $S=S\left(d, G_{i}, \gamma_{i}\right)$. A subset $A$ of $G=G_{0} \times G_{1} \times \ldots \times G_{d-1}$ which satisfies the conditions

(i) $A=A_{0} \times A_{1} \times \ldots \times A_{d-1}$ for some $A_{i} \subseteq G_{i}, i=0, \ldots, d-1$,

(ii) $A_{i} \unlhd G_{i}, i=0, \ldots, d-1$,

(iii) $A_{d-1} \gamma_{d-1} \subseteq A_{0}$ and $A_{i} \gamma_{i} \subseteq A_{i+1}, i=0, \ldots, d-2$,

is called a $\gamma$-admissible subset of $G$.

$\Gamma^{*}$ denotes the set of the $\gamma$-admissible subsets of $G$ satisfying the condition

(iv) $\operatorname{rad} A=A$ where $\operatorname{rad} A=\operatorname{rad} A_{0} \times \ldots \times \operatorname{rad} A_{d-1}$ and

$$
\operatorname{rad} A_{i}=\left\{a_{i} \in G_{i} \mid a_{i} \alpha_{i}^{n} \in A_{i} \text { for some nonnegative integer } n\right\} \text {. }
$$

Lemma 2.6 ([2], Lemma 3.2, Lemma 3.4 and Lemma 3.5). Let $S=S\left(d, G_{i}, \gamma_{i}\right)$ and let $\rho$ be a congruence on $S$ such that $\rho \in[\sigma, \sigma \vee \mathbb{H}]$. Put $A^{\rho}=A_{0}^{\rho} \times \ldots \times A_{d-1}^{\rho}$ where $A_{i}^{\rho}=\left\{a_{i} \in G_{i} \mid\left(0, a_{i}, 0\right) \in \operatorname{ker} \rho\right\}$. Then the following conditions hold.

(i) $A^{\rho} \in \Gamma^{*}$.

(ii) $\operatorname{ker} \rho=\left\{\left(m, a_{i}, m\right) \mid m \in \mathbb{N}, a_{i} \in A_{i}^{\rho}, i=0, \ldots, d-1\right\}$.

(iii) Let $x=\left(m, g_{i}, n\right), y=\left(p, h_{j}, q\right)$ be two elements of $S$, then we have $x \rho y$ if and only if $m-n=p-q$ and $\left(g_{i} \alpha_{u, w}\right)\left(h_{j}^{-1} \alpha_{v, w}\right) \in A_{k}^{\rho}$ where $u=n d+i, v=q d+j, w=$ $\max \{u, v\}, k=\bar{w}$.

Conversely, for every $A \in \Gamma^{*}$, the relation $\rho$ defined by (iii) is a congruence on $S$ belonging to $[\sigma, \sigma \vee \mathbb{H}]$ such that $A^{\rho}=A$.

Remark 2.7. By Lemma 2.6 it follows that a group congruence $\rho$ contained in $\sigma \vee H$ is completely determined by the subset $A^{\rho}$ of $G$. Hence, by Lemma $2.3, \lambda$ can be described by means of $A^{\lambda}$.

Remark 2.8. We recall that for every two congruences $\rho$ and $\tau$ on an inverse semigroup, we have $\rho \leq \tau$ if and only if $\operatorname{tr} \rho \leq \operatorname{tr} \tau$ and $\operatorname{ker} \rho \subseteq \operatorname{ker} \tau$. Hence, if $S=S\left(d, G_{i}, \gamma_{i}\right)$ and $\rho, \tau \in[\sigma, \sigma \vee \mathbb{H}]$ then $\rho \leq \tau$ if and only if $A^{\rho} \subseteq A^{\tau}$.

Definition 2.9. Let $H$ be a group and $\phi$ an endomorphism of $H$. For every $a, b \in H$ we call

$$
\left(a \phi^{r}\right)\left(b \phi^{s}\right)\left(a^{-1} \phi^{t}\right)\left(b^{-1} \phi^{u}\right) \quad(r, s, t, u \in \mathbb{N})
$$

a $\phi$-commutator of $a$ and $b$, and, if it is unambiguous, we put

$$
\left(a \phi^{r}\right)\left(b \phi^{s}\right)\left(a^{-1} \phi^{\prime}\right)\left(b^{-1} \phi^{u}\right)=[a, b]_{\phi} .
$$

We denote by $H_{\phi}^{\prime}$ the subgroup of $H$ generated by the $\phi$-commutators of $H$ and we call it the $\phi$-derivate of $H$.

Lemma 2.10. The following properties hold.

(i) $H_{\phi}^{\prime} \supseteq H^{\prime}$ where $H^{\prime}$ indicates the derivate of the group $H$.

(ii) If $g \phi^{k} \in H_{\phi}^{\prime}$ for some non negative integer $k$, then $g \in H_{\phi}^{\prime}$.

(iii) $H_{\phi}^{\prime} \unlhd H$. 
Proof. Property (i) is obvious, property (ii) easily follows because $g\left(g^{-1} \phi^{k}\right)$ is a $\phi$-commutator of $g$ and of the identity of $H$. To prove property (iii), let $a, b, g \in H$ and consider a $\phi$-commutator

If $t<r$,

$$
[a, b]_{\phi}=\left(a \phi^{r}\right)\left(b \phi^{s}\right)\left(a^{-1} \phi^{\prime}\right)\left(b^{-1} \phi^{u}\right)
$$

$$
\begin{aligned}
g[a, b]_{\phi} g^{-1} & =g\left(\left(a \phi^{t}\right) \phi^{r-t}\right) g^{-1}\left(a^{-1} \phi^{\prime}\right)\left(a \phi^{\prime}\right) g\left(b \phi^{s}\right) g g^{-1}\left(a^{-1} \phi^{t}\right) g^{-1}\left(b^{-1} \phi^{s}\right)\left(b \phi^{s}\right) g\left(b^{-1} \phi^{u}\right) g^{-1} \\
& =\left[g, a \phi^{\prime}\right]_{\phi}\left[\left(a \phi^{\prime}\right) g,\left(b \phi^{s}\right) g\right]_{\phi}[b, g]_{\phi} .
\end{aligned}
$$

If, on the contrary $t \geq r$,

$$
\begin{aligned}
g[a, b]_{\phi} g^{-1} & =g\left(a \phi^{r}\right)\left(b \phi^{s}\right)\left(\left(a^{-1} \phi^{r}\right) \phi^{t-r}\right)\left(g^{-1} \phi^{t-r}\right) b^{-1} b\left(g \phi^{t-r}\right)\left(b^{-1} \phi^{u}\right) g^{-1} \\
& =\left[g\left(a \phi^{r}\right), b\right]_{\phi}[b, g]_{\phi} .
\end{aligned}
$$

Lemma 2.11. Let $S=S\left(d, G_{i}, \gamma_{i}\right)$ and $\lambda$ its least commutative congruence. Then $A^{\lambda}=G_{\alpha}^{\prime}$ where $G_{\alpha}^{\prime}=\left(G_{0}\right)_{\alpha_{0}}^{\prime} \times \ldots \times\left(G_{d-1}\right)_{\alpha_{d-1}}^{\prime}$.

Proof. First we prove that $G_{\alpha}^{\prime} \in \Gamma^{*}$. In fact condition (i) of Definition 2.5 obviously holds; conditions (ii) and (iv) follow by Lemma 2.10. Moreover $\alpha_{i} \gamma_{i}=\gamma_{i} \alpha_{i+1}$ for every $i=0, \ldots, d-2, \alpha_{d-1} \gamma_{d-1}=\gamma_{d-1} \alpha_{0}$. Let $0 \leq j \leq d-1$. For every $a_{j}, b_{j} \in G_{j}$ and for every $\alpha_{j}$-commutator of $a_{j}, b_{j}$ we have

$$
\left[a_{d-1}, b_{d-1}\right]_{\alpha_{d-1}} \gamma_{d-1}=\left[a_{d-1} \gamma_{d-1}, b_{d-1} \gamma_{d-1}\right]_{\alpha_{0}}
$$

and $\left[a_{j}, b_{j}\right]_{\alpha_{j}} \gamma_{j}=\left[a_{j} \gamma_{j}, b_{j} \gamma_{j}\right]_{\alpha_{j+1}}$ with $j \leq d-2$, i.e. condition (iii) of Definition 2.5 holds. Now, let $\rho$ be the group congruence induced by $G_{\alpha}^{\prime}$ following Lemma 2.6. We will show that $\rho$ is commutative, i.e. that for every $x, y \in S$ we have $x y \rho y x$. Put then

$$
x=\left(m, g_{i}, n\right), \quad y=\left(p, h_{j}, q\right),\left(g_{i} \in G_{i}, h_{j} \in G_{j} ; 0 \leq i, j \leq d-1 ; m, n, p, q \in \mathbb{N}\right),
$$

and

$$
x y=\left(m+p-r,\left(g_{i} \alpha_{u, w}\right)\left(h_{j} \alpha_{v, w}\right), q+n-r\right)
$$

$$
y x=\left(p+m-s,\left(h_{j} \alpha_{a, c}\right)\left(g_{i} \alpha_{b, c}\right), q+n-s\right)
$$

where $r=\min \{n, p\}, s=\min \{q, m\}, u=n d+i, v=p d+j, w=\max \{u, v\}, a=q d+j$, $b=m d+i, c=\max \{a, b\}$.

Obviously condition

$$
(m+p-r)-(q+n-r)=(p+m-s)-(q+n-s)
$$

holds. Now consider the element

with

$$
g=\left(\left(\left(g_{i} \alpha_{u, w}\right)\left(h_{j} \alpha_{v, w}\right)\right) \alpha_{l, t}\right)\left(\left(\left(h_{j} \alpha_{a, c}\right)\left(g_{i} \alpha_{b, c}\right)\right)^{-1} \alpha_{k, t}\right)
$$

$$
l=(n+q-r) d+\bar{w}, \quad k=(q+n-s) d+\bar{c}, \quad t=\max \{l, k\}
$$

We have

$$
\begin{aligned}
g & =\left(\left(g_{i} \alpha_{u, w}\right) \alpha_{l, t}\right)\left(\left(h_{j} \alpha_{v, w}\right) \alpha_{l, t}\right)\left(\left(g_{i}^{-1} \alpha_{b, c}\right) \alpha_{k, t}\right)\left(\left(h_{j}^{-1} \alpha_{a, c}\right) \alpha_{k, t}\right) \\
& \left.=\left(\left(g_{i} \alpha_{i, z}\right) \alpha_{z}^{i_{1}}\right)\left(\left(h_{j} \alpha_{j, z}\right) \alpha_{z}^{i_{2}}\right)\left(\left(g_{i} \alpha_{i, z}\right)^{-1} \alpha_{z}^{i_{3}}\right)\left(\left(h_{j} \alpha_{j, z}\right)^{-1}\right) \alpha_{z}^{i_{4}}\right)
\end{aligned}
$$


where, if $\bar{t} \geq \max \{i, j\}, \quad z=\bar{t}$, otherwise $z=\bar{t}+d$ and $i_{1}, i_{2}, i_{3}, i_{4}$ are suitable non negative integers (Remark 2.1). By (2) it follows that $g \in\left(G_{i}\right)_{\alpha_{i}^{-}}^{\prime}$; hence by (1) and by condition (iii) of Lemma 2.6, $x y \rho y x$.

Now, let $\tau$ be a commutative congruence on $S$; we shall prove that $\rho \leq \tau$. The congruence $\tau^{\prime}=\tau \wedge(\sigma \vee \mathbb{H})$ is commutative; hence it is a group congruence by Corollary 2.4. Let $A^{\boldsymbol{r}^{\prime}}$ be the $\gamma$-admissible subgroup of $G$ induced by $\tau^{\prime}$ following Lemma 2.6 and let

$$
\left(g_{i} \alpha_{i}^{p}\right)\left(h_{i} \alpha_{i}^{n}\right)\left(g_{i}^{-1} \alpha_{i}^{q}\right)\left(h_{i}^{-1} \alpha_{i}^{m}\right) \quad\left(i=0, \ldots, d-1 ; g_{i}, h_{i} \in G_{i} ; p, n, q, m \in \mathbb{N}\right\}
$$

be an $\alpha_{i}$-commutator of $G_{i}$. Put $x=\left(m+k, g_{i}, n+k\right), y=\left(p+k, h_{i}, q+k\right)$ where $k=\min \{r, s\}, r=\min \{p, n\}, s=\min \{q, m\}$. Since $\tau^{\prime}$ is a commutative congruence we have $x y \tau^{\prime} y x$, hence recalling condition (iii) of Lemma 2.6 and Remark 2.2 it follows that

$$
\left[\left(g_{i} \alpha_{i}^{p+k-t}\right)\left(h_{i} \alpha_{i}^{n+k-t}\right)\right] \alpha_{u, w}\left[\left(g_{i}^{-1} \alpha_{i}^{q+k-j}\right)\left(h_{i}^{-1} \alpha_{i}^{m+k-j}\right)\right] \alpha_{v, w} \in A_{i}^{\tau^{\prime}}
$$

where $\quad t=\min \{n+k, p+k\}, \quad j=\min \{q+k, m+k\}, \quad u=(n+q+2 k-t) d+i$, $v=(n+q+2 k-j) d+j$ and $w=\max \{u, v\}$, whence

$$
\left(g_{i} \alpha_{i}^{p}\right)\left(h_{i} \alpha_{i}^{n}\right)\left(g_{i}^{-1} \alpha_{i}^{q}\right)\left(h_{i}^{-1} \alpha_{i}^{m}\right) \in A_{i}^{\tau^{\prime}}
$$

thus $\left(G_{i}\right)_{\alpha_{i}}^{\prime} \subseteq A_{i}^{\tau^{\prime}}$ and $G_{\alpha}^{\prime} \subseteq A^{\tau^{\prime}}$. So $\rho \leq \tau^{\prime} \leq \tau$, hence $\rho=\lambda$.

By previous Lemmas we can deduce the following description of the least commutative congruence for a simple regular $\omega$-semigroup.

THEOREM 2.12. Let $S=S\left(d, G_{i}, \gamma_{i}\right)$ be a simple regular $\omega$-semigroup and $\lambda$ its least commutative congruence. Then

and

$$
\left(m, g_{i}, n\right) \lambda\left(p, h_{j}, q\right) \text { if and only if } m-n=p-q
$$

with

$$
\left(g_{i} \alpha_{u, w}\right)\left(h_{j}^{-1} \alpha_{v, w}\right) \in\left(G_{z}\right)_{\alpha_{2}}^{\prime}
$$

$$
u=n d+i, \quad v=q d+j, \quad w=\max \{u, v\}, \quad z=\bar{w} .
$$

REMARK 2.13. $\lambda$ is a group congruence such that

$$
\operatorname{ker} \lambda=\left\{\left(m, g_{i}, m\right) \mid m \in \mathbb{N}, g_{i} \in\left(G_{i}\right)_{\alpha_{i}}^{\prime} ; i=0, \ldots, d-1\right\} \text {. }
$$

Clearly $\operatorname{ker} \lambda$ is an $\omega$-chain of groups.

THEOREM 2.14. Let $S=S\left(d, G_{i}, \gamma_{i}\right)$ be a simple regular $\omega$-semigroup. Denote by $\mathscr{G}$ the direct product $G_{0} /\left(G_{0}\right)_{\alpha_{0}}^{\prime} \times \ldots \times G_{d-1} /\left(G_{d-1}\right)_{\alpha_{d-1}}^{\prime}$ and consider the subgroup $K$ of $\mathscr{G}$ defined by

$$
\mathscr{K}=\left\{\left(g_{i} \alpha_{i, d}\left(G_{0}\right)_{\alpha_{0}}^{\prime}, \ldots, g_{i} \alpha_{i, d+s}\left(G_{s}\right)_{\alpha_{s}}^{\prime}, \ldots, g_{i} \alpha_{i, 2 d-1}\left(G_{d-1}\right)_{\alpha_{d-1}}^{\prime}\right) \mid g_{i} \in G_{i} ; i, s=0, \ldots, d-1\right\} \text {. }
$$

The mapping $f$ of $S / \lambda$ onto $\mathscr{K} \times \mathbb{Z}$ defined by

$$
f:\left(m, g_{i}, n\right) \lambda \rightarrow\left(\left(g_{i} \alpha_{i, d}\left(G_{0}\right)_{\alpha_{0}}^{\prime}, \ldots, g_{i} \alpha_{i, 2 d-1}\left(G_{d-1}\right)_{\alpha_{d-1}}^{\prime}\right), m-n\right)
$$

is an isomorphism.

Proof. Consider $\left(p, h_{j}, q\right) \lambda \in S / \lambda$ and its image

$$
\left(\left(h_{j} \alpha_{j, d}\left(G_{0}\right)_{\alpha_{0}}^{\prime}, \ldots, h_{j} \alpha_{j, 2 d-1}\left(G_{d-1}\right)_{\alpha_{d-1}}^{\prime}\right), p-q\right)
$$


First we prove that $\left(m, g_{i}, n\right) \lambda=\left(p, h_{j}, q\right) \lambda$ iff

$$
\begin{aligned}
\left(\left(g_{i} \alpha_{i, d}\left(G_{0}\right)_{\alpha_{0}}^{\prime}, \ldots, g_{i} \alpha_{i, 2 d-1}\left(G_{d-1}\right)_{\alpha_{d-1}}^{\prime}\right), m-n\right) & \\
= & \left(\left(h_{j} \alpha_{j, d}\left(G_{0}\right)_{\alpha_{0}}^{\prime}, \ldots, h_{j} \alpha_{j, 2 d-1}\left(G_{d-1}\right)_{\alpha_{d-1}}^{\prime}\right), p-q\right) .
\end{aligned}
$$

In fact suppose $\left(m, g_{i}, n\right) \lambda\left(p, h_{j}, q\right)$; then from Theorem 2.12 it follows that $m-n=$ $p-q$ and $g_{i} \alpha_{u, w} h_{j}^{-1} \alpha_{v, w} \in\left(G_{\bar{w}}\right)_{\alpha_{\tilde{w}}}^{\prime}$ with $u=n d+i, v=q d+j$ and $w=\max \{u, v\}$. Hence, by Remark $2.1\left(g_{i} \alpha_{i, \tilde{w}+d}\right) \alpha_{\bar{w}}^{i_{1}}\left(h_{j}^{-1} \alpha_{j, \tilde{w}+d}\right) \alpha_{w}^{i_{w}} \in\left(G_{\bar{w}}\right)_{\alpha_{\tilde{w}}}^{\prime}$ for some nonnegative integers $i_{1}, i_{2}$ and, since we have

$$
\left(g_{i} \alpha_{i, \bar{w}+d}\right) \alpha_{\bar{w}}^{i_{1}}\left(h_{j}^{-1} \alpha_{j, \bar{w}+d}\right) \alpha_{\bar{w}}^{i_{2}}\left(g_{i}^{-1} \alpha_{i, \bar{w}+d}\right)\left(h_{j} \alpha_{j, \bar{w}+d}\right) \in\left(G_{\bar{w}}\right)_{\alpha_{\bar{w}}}^{\prime}
$$

we deduce $\left(g_{i}^{-1} \alpha_{i, \bar{w}+d}\right)\left(h_{j} \alpha_{j, \bar{w}+d}\right) \in\left(G_{\bar{w}}\right)_{\alpha_{\bar{w}}{ }^{\prime}}^{\prime}$. Hence for every nonnegative integer $k$

$$
\left[\left(g_{i}^{-1} \alpha_{i, \bar{w}+d}\right)\left(h_{j} \alpha_{j, \bar{w}+d}\right)\right] \alpha_{\bar{w}+d, \bar{w}+\bar{k}+d} \in\left(G_{\bar{w}}\right)_{\alpha_{\bar{w}}}^{\prime} \alpha_{\bar{w}+d, \bar{w}+\bar{k}+d} \subseteq\left(G_{\bar{w}+k}\right)_{\alpha_{\bar{w}+k}}^{\prime}
$$

and, by Remark 2.1,

$$
\left[\left(g_{i}^{-1} \alpha_{i, \overline{w+k}+d}\right)\left(h_{j} \alpha_{j, \overline{w+k}+d}\right)\right] \alpha_{\frac{w+k}{i_{3}}+d}^{i_{3}} \in\left(G_{\overline{w+k}}\right)_{\alpha_{\overline{w+k}}}^{\prime}
$$

for some nonnegative integer $i_{3}$. So (ii) of Lemma 2.10 gives

$$
\left(g_{i}^{-1} \alpha_{i, \overline{w+k}+d}\right)\left(h_{j} \alpha_{j, \overline{w+k}+d}\right) \in\left(G_{\overline{w+k}}\right)_{\alpha_{w+k}}^{\prime}
$$

whence, since $k$ is an arbitrary nonnegative integer, $\left(g_{i}^{-1} \alpha_{i, t+d}\right)\left(h_{j} \alpha_{j, t+d}\right) \in\left(G_{t}\right)_{\alpha_{t}}^{\prime}$ for every integer $t$ with $0 \leq t \leq d-1$. Thus, we deduce that $\left(m, g_{i}, n\right) \lambda\left(p, h_{j}, q\right)$ implies $h_{j} \alpha_{j, t+d} \in$ $g_{i} \alpha_{i, t+d}\left(G_{t}\right)_{\alpha_{t}}^{\prime}$ for every $t=0, \ldots, d-1$ and $m-n=p-q$ whence

$$
\begin{aligned}
\left(\left(g_{i} \alpha_{i, d}\left(G_{0}\right)_{\alpha_{0}}^{\prime}, \ldots, g_{i} \alpha_{i, 2 d-1}\left(G_{d-1}\right)_{\alpha_{d-1}}^{\prime}\right), m-n\right) & =\left(\left(h_{j} \alpha_{j, d}\left(G_{0}\right)_{\alpha_{0}}^{\prime}, \ldots, h_{j} \alpha_{j, 2 d-1}\left(G_{d-1}\right)_{\alpha_{d-1}}^{\prime}\right), p-q\right) .
\end{aligned}
$$

Conversely, let

$$
\begin{aligned}
\left(\left(g_{i} \alpha_{i, d}\left(G_{0}\right)_{\alpha_{0}}^{\prime}, \ldots, g_{i} \alpha_{i, 2 d-1}\left(G_{d-1}\right)_{\alpha_{d-1}}^{\prime}\right)\right. & , m-n) \\
& =\left(\left(h_{j} \alpha_{j, d}\left(G_{0}\right)_{\alpha_{0}}^{\prime}, \ldots, h_{j} \alpha_{j, 2 d-1}\left(G_{d-1}\right)_{\alpha_{d-1}}^{\prime}\right), p-q\right) .
\end{aligned}
$$

This implies $m-n=p-q$ and $g_{i}^{-1} \alpha_{i, \bar{k}+d} h_{j} \alpha_{j, \bar{k}+d} \in\left(G_{\bar{k}}\right)_{\alpha_{\bar{k}}}^{\prime}$ for every nonnegative integer $k$ whence

$$
\left(g_{i} \alpha_{i, \bar{k}+d}\right) \alpha_{\bar{k}}^{j_{1}}\left(h_{j}^{-1} \alpha_{j, \bar{k}+d}\right) \alpha_{\bar{k}}^{j_{2}} \in\left(G_{\bar{k}}\right)_{\alpha_{\bar{k}}}^{\prime}
$$

for every nonnegative integers $j_{1}, j_{2}$. Let $u=n d+i, v=q d+j$ and $w=\max \{u, v\}$. Then for every $j_{1}, j_{2}$ such that

we have

$$
\min \left\{\bar{k}+\left(j_{1}+n+1\right) d, \bar{k}+\left(j_{2}+q+1\right) d\right\} \geq w,
$$

and

$$
\alpha_{i, \bar{k}+d} \alpha_{\bar{k}}^{j_{1}}=\alpha_{i, \bar{k}+\left(1+j_{1}\right) d}=\alpha_{u, \bar{k}+\left(j_{1}+n+1\right) d}=\alpha_{u, w} \alpha_{w, \bar{k}+\left(j_{1}+n+1\right) d}
$$

$$
\alpha_{j, \bar{k}+d} \alpha_{\bar{k}}^{j_{2}}=\alpha_{v, \bar{k}+\left(j_{2}+q+1\right) d}=\alpha_{v, w} \alpha_{w, \bar{k}+\left(j_{2}+q+1\right) d} .
$$

Now let $w=\bar{w}+t d$; if $n \geq q$, choosing $\bar{k}=\bar{w}$ and $j_{1}=t, j_{2}=t+n-q$, we have

$$
\bar{k}+\left(j_{1}+n+1\right) d=\bar{w}+t d+n d+d=w+(n+1) d=\bar{k}+\left(j_{2}+q+1\right) d .
$$


Hence, by Remark 2.1, $\left(g_{i} \alpha_{u, w} h_{j}^{-1} \alpha_{v, w}\right) \alpha_{\bar{w}}^{n+1} \in\left(G_{\bar{w}}\right)_{\alpha_{\bar{w}}}^{\prime}$. Analogously, if $n<q$, putting $j_{1}=t+q-n, j_{2}=t$, we obtain

$$
\left(g_{i} \alpha_{u, w} h_{j}^{-1} \alpha_{v, w}\right) \alpha_{\bar{w}}^{q+1} \in\left(G_{\bar{w}}\right)_{\alpha_{\bar{w}}}^{\prime}
$$

In both the cases from (ii) of Lemma 2.10 it follows that $\left(g_{i} \alpha_{u, w} h_{j}^{-1} \alpha_{v, w}\right) \in\left(G_{\bar{w}}\right)_{\alpha_{\tilde{w}}}^{\prime}$ with $u=n d+i, v=q d+j$ and $w=\max \{u, v\}$, so we have $\left(m, g_{i}, n\right) \lambda=\left(p, h_{j}, q\right) \lambda$. Thus $f$ is well defined and injective.

The mapping $f$ is obviously onto and finally it is a homomorphism; in fact consider

$$
\left[\left(m, g_{i}, n\right) \lambda\right]\left[\left(p, h_{j}, q\right) \lambda\right]=\left(m+p-r,\left(g_{i} \alpha_{n d+i, w}\right)\left(h_{j} \alpha_{p d+j, w}\right), n+q-r\right) \lambda,
$$

with $w=\max (n d+i, p d+j), r=\min (n, p)$.

$$
\begin{aligned}
f\left(\left(m+p-r,\left(g_{i} \alpha_{n d+i, w}\right)\right.\right. & \left.\left.\left(h_{j} \alpha_{p d+i, w}\right), n+q-r\right) \lambda\right) \\
= & \left(\left(\left(g_{i} \alpha_{n d+i, w} h_{j} \alpha_{p d+j, w}\right) \alpha_{\bar{w}, d}\left(G_{0}\right)_{\alpha_{0}}^{\prime}, \ldots,\right.\right. \\
& \left.\left.\quad\left(g_{i} \alpha_{n d+i, w} h_{j} \alpha_{p d+j, w}\right) \alpha_{\bar{w}, 2 d-1}\left(G_{d-1}\right)_{\alpha_{d-1}}^{\prime}\right), m+p-n-q\right) .
\end{aligned}
$$

Also

$$
\begin{array}{r}
\left(\left(g_{i} \alpha_{i, d}\left(G_{0}\right)_{\alpha_{0}}^{\prime} \ldots, g_{i} \alpha_{i, 2 d-1}\left(G_{d-1}\right)_{\alpha_{d-1}}^{\prime}, m-n\right)\left(\left(h_{j} \alpha_{j, d}\left(G_{0}\right)_{\alpha_{0}}^{\prime}, \ldots, h_{j} \alpha_{j, 2 d-1}\left(G_{d-1}\right)_{\alpha_{d-1}}^{\prime}\right), p-q\right)\right. \\
=\left(\left(g_{i} \alpha_{i, d} h_{j} \alpha_{j, d}\left(G_{0}\right)_{\alpha_{0}}^{\prime}, \ldots, g_{i} \alpha_{i, 2 d-1} h_{j} \alpha_{j, 2 d-1}\left(G_{d-1}\right)_{\alpha_{d-1}}^{\prime}\right), m-n+p-q\right) .
\end{array}
$$

Moreover

$$
g_{i} \alpha_{i, d+\bar{h}} h_{j} \alpha_{j, d+\bar{h}}\left(h_{j}^{-1} \alpha_{p d+j, w}\right) \alpha_{\bar{w}, d+\bar{h}}\left(g_{i}^{-1} \alpha_{n d+i, w}\right) \alpha_{\bar{w}, d+\bar{h}} \in\left(G_{\bar{h}}\right)_{\alpha_{\bar{h}}}^{\prime}
$$

in fact, if $w=p d+j, \quad\left(h_{j} \alpha_{p d+j, w}\right) \alpha_{\tilde{w}, d+\bar{h}}=h_{j} \alpha_{j, d+\bar{h}} \quad$ and, by Remark 2.1, $g_{i} \alpha_{i, d+\bar{h}}\left(g_{i}^{-1} \alpha_{n d+i, w}\right) \alpha_{\bar{w}, d+\bar{h}} \in\left(G_{\bar{h}}\right)_{\alpha_{\bar{h}}}^{\prime}$; if $w=n d+i, g_{i} \alpha_{i, d+\bar{h}}=\left(g_{i} \alpha_{n d+i, w}\right) \alpha_{\bar{w}, d+\bar{h}}$ and by Remark $2.1, h_{j} \alpha_{j, d+\bar{h}}\left(h_{j}^{-1} \alpha_{p d+j, w}\right) \alpha_{\bar{w}, d+\bar{h}} \in\left(G_{\bar{h}}\right)_{\alpha_{\bar{h}}}^{\prime}$, hence the result follows because $\left(G_{\bar{h}}\right)_{\alpha_{\bar{h}}}^{\prime}$ is a normal subgroup of $G_{h}$. Thus, we have

$$
\left.f\left(\left[\left(m, g_{i}, n\right) \lambda\right]\left(p, h_{j}, q\right) \lambda\right]\right)=f\left(\left(m, g_{i}, n\right) \lambda\right) f\left(\left(p, h_{j}, q\right) \lambda\right)
$$

When $d=1, S$ is a bisimple $\omega$-semigroup, usually denoted by $S=S(G, \alpha)$. Thus we have the following result.

COROLLARY 2.15. The kernel of the least commutative congruence $\lambda$ on a bisimple $\omega$-semigroup $S$ is the commutator subsemigroup $S^{\prime}$, which is an $\omega$-chain of groups $S_{i}$ isomorphic to $\left(G^{\prime}\right)_{\alpha}$ for every integer $i$. The semigroup $S / \lambda$ is a commutative group which is isomorphic to the direct product $G /\left(G^{\prime}\right)_{\alpha} \times \mathbb{Z}$.

Proof. Let $x$ be an element of ker $\lambda$, thus from Remark 2.13 it follows that $x=\left(m, a \alpha^{r} b \alpha^{s} a^{-1} \alpha^{t} b^{-1} \alpha^{v}, m\right)=\left\{\left(m, a \alpha^{r}, m\right)\left(m, b \alpha^{s}, m\right)\left(m, a^{-1} \alpha^{r}, m\right)\left(m, b^{-1} \alpha^{s}, m\right)\right\}$ . $\left\{\left(m, b \alpha^{s}, m\right)\left(m, a \alpha^{r}, m\right)\left(m, a^{-1} \alpha^{\prime}, m\right)\left(m, b^{-1} \alpha^{s}, m\right)\right\}\left\{\left(m, b \alpha^{s}, m\right)\left(m, b^{-1} \alpha^{\nu}, m\right)\right\}$.

Moreover, denoting by $e$ the identity of $G$, for every $b \in G$ and $s, v$ nonnegative integers, if $s \leq v$ we have

$$
\left(m, b \alpha^{s}, m\right)\left(m, b^{-1} \alpha^{v}, m\right)=\left[\left(m, b \alpha^{s}, 0\right),(0, e, v-s)\right] \in S^{\prime}
$$

and if $s>v$ we have

$$
\begin{aligned}
\left(m, b \alpha^{s}, m\right)\left(m, b^{-1} \alpha^{v}, m\right) & =\left\{\left(m, b \alpha^{v}, m\right)\left(m, b^{-1} \alpha^{s}, m\right)\right\}^{-1} \\
& =\left[\left(m, b \alpha^{v}, 0\right),(0, e, s-v)\right]^{-1} \in S^{\prime}
\end{aligned}
$$


Now, we consider

$$
\left(m, b \alpha^{s}, m\right)\left(m, a \alpha^{r}, m\right)\left(m, a^{-1} \alpha^{t}, m\right)\left(m, b^{-1} \alpha^{s}, m\right)=\left(m, b \alpha^{s} a \alpha^{r} a^{-1} \alpha^{t} b^{-1} \alpha^{s}, m\right) .
$$

If $r \leq t$, by a simple calculus, we have

$$
\begin{aligned}
\left(m, b \alpha^{s} a \alpha^{r} a^{-1} \alpha^{t} b^{-1} \alpha^{s}, m\right)= & {\left[\left(m, b \alpha^{s} a \alpha^{r} b^{-1} \alpha^{s}, 0\right),\left(0, b \alpha^{s} b^{-1} \alpha^{s+t-r}, t-r\right)\right] } \\
& .\left(m, b \alpha^{s+m} b^{-1} \alpha^{s+m+t-r}, m\right)\left(m, b \alpha^{s+t-r} b^{-1} \alpha^{s}, m\right) \in S^{\prime},
\end{aligned}
$$

if $r>t$

$$
\left(m, b \alpha^{s} a \alpha^{\prime} a^{-1} \alpha^{t} b^{-1} \alpha^{s}, m\right)=\left(m, b \alpha^{s} a \alpha^{\prime} a^{-1} \alpha^{\prime} b^{-1} \alpha^{s}, m\right)^{-1} \in S^{\prime} .
$$

Hence $x \in S^{\prime}$ and $\operatorname{ker} \lambda=S^{\prime}$, moreover, from Remark 2.13 it follows that $S^{\prime}$ is an $\omega$-chain of groups $S_{i}$ isomorphic to $\left(G^{\prime}\right)_{\alpha}$. Finally from Lemma 2.3 it follows that $\lambda$ is a group congruence and from Theorem 2.14 we deduce that $S / \lambda$ is isomorphic to the direct product $\left[G /\left(G^{\prime}\right)_{\alpha}\right] \times \mathbb{Z}$.

For a direct proof see [11], Theorem 2.5.

3. Solvability of simple regular $\omega$-semigroups. In $[10]$ the following definition was introduced for inverse semigroups:

Definition 3.1. Let $S$ be an inverse semigroup. Denote $\delta_{0}(S)=S, \lambda_{0}=\omega_{s}$, the universal congruence on $S$, and for $i \geq 1$, let $\lambda_{i, S}$ (or simply $\lambda_{i}$ ) be the least commutative congruence on $\delta_{i-1}(S)=\operatorname{ker} \lambda_{i-1}$ (trivially $\left.\lambda_{1}=\gamma\right)$. S is called solvable of solvability class $c$ or $c$-solvable if $c$ is the least index $i$ such that $\lambda_{c}=\mathrm{id}_{\delta_{c-1}(S)}$, the identity map on $\delta_{c-1}(S)$.

LEMMA 3.2 ([10], 3.3). $S$ is solvable of class $c$ if and only if $c$ is the least index $i$ such that $\delta_{i-1}(S)$ is commutative.

Since any simple regular $\omega$-semigroup is trivially inverse, it makes sense to ask about its solvability. We want to prove that there is a strict connection between the solvability of $S$ and that of the groups $G_{i}$.

We remark that a simple regular $\omega$-semigroup is a Bruck-Reilly semigroup over $T$ where $T$ is a chain $G_{0}>G_{1}>\ldots>G_{d-1}$ of groups [6, Structure Theorem], hence we state the result for Bruck-Reilly semigroups. We recall the following definition.

Definition 3.3. Let $T$ be a monoid, $\alpha$ be a homomorphism of $T$ into its group of units. The Bruck-Reilly semigroup over $T$ is the semigroup $B(T, \alpha)$ of the triplets $(m, a, n): m, n$ are nonnegative integers, $a \in T$ and the multiplication is defined as follows:

$$
(m, a, n)(p, b, q)=\left(m+p-r,\left(a \alpha^{p-r}\right)\left(b \alpha^{n-r}\right), n+q-r\right)
$$

where $r=\min (n, p)$ and $\alpha^{0}$ is the identity map on $T$.

It is well-known (see [9], e.g.) that $B(T, \alpha)$ is a simple monoid for each $T$ and $\alpha$, and that it is inverse if and only if $T$ is inverse.

THEOREM 3.4. Let $S=B(T, \alpha)$ be a Bruck-Reilly semigroup over an inverse monoid $T$. Then $S$ is solvable if and only if $T$ is solvable. If $S$ is solvable of class $n$ then $T$ is solvable of class $n$ or $n-1$.

Proof. If $S$ is a solvable semigroup of class $n$, then, by Theorem 3.5 of [10], $T$ is immediately seen to be solvable, and its solvability class is less than or equal to $n$, since $T$ is (isomorphic to) a subsemigroup of $S$. 
To prove that the condition is sufficient, consider firstly a commutator of elements of $S:$

$$
c=[(m, a, n),(p, b, q)] .
$$

Let $r=\min (n, p), t=\min (m, q), v=\min (n+q-r, n+q-t)$. Then

$$
\begin{array}{r}
c=\left(m+p-r+n+q-t-v,\left(a^{-1} \alpha^{p-r} b^{-1} \alpha^{n-r}\right) \alpha^{n+q-t-v}\left(a \alpha^{q-t} b \alpha^{m-r}\right) \alpha^{n+q-r-v}, m\right. \\
+p-t+n+q-r-v) .
\end{array}
$$

Remark that:

$$
n+q-r-t-v=\left\langle\begin{array}{l}
=-t \Leftrightarrow v=n+q-r \Leftrightarrow t \leq r \Leftrightarrow t=\min (m, q) \leq \min (n, p) \\
=-r \Leftrightarrow v=n+q-t \Leftrightarrow r \leq t \Leftrightarrow r=\min (n, p) \leq \min (m, q)
\end{array} .\right.
$$

If we denote $k=\min (m, n, p, q)$, then we have proved that:

$$
c=[(m, a, n),(p, b, q)]=\left(m+p-k, a \alpha^{p-k} b \alpha^{n-k} a^{-1} \alpha^{q-k} b^{-1} \alpha^{m-k}, m+p-k\right) .
$$
then:

Since the product of two elements $(m, a, m)$ and $(p, b, p)$ is again of type $(n, x, n)$

$$
(m, a, n) \in S^{\prime} \quad \text { implies that } m=n \text {. }
$$

Let

$$
(m, a, n)(p, e, p)=(m+p-\min (n, p), \ldots, n+p-\min (n, p)),
$$

where $(p, e, p) \in E_{S}$. Such a product belongs to $S^{\prime}$ only if $m+p-\min (n, p)=n+p-$ $\min (n, p)$, i.e. Only if $m=n$. Then

$$
(m, a, n) \in \delta(S) \text { implies that } m=n \text {. }
$$

Now, since $(m, a, m) \in \delta(S)$ implies trivially that $a \in T=\delta_{0}(T)$, suppose, by induction, that we have proved:

$$
\text { for } i \geq 2,(m, a, m) \in \delta_{i-1}(S) \text { implies that } a \in \delta_{i-2}(T) \text {. }
$$

Consider $c=[(p, a, p),(q, b, q)] \in\left(\delta_{i-1}(S)\right)^{\prime}$; for $k=\min (p, q)$, one has:

$$
\begin{aligned}
c & =\left(p+q-k, a \alpha^{q-k} b \alpha^{p-k} a^{-1} \alpha^{q-k} b^{-1} \alpha^{p-k}, p+q-k\right) \\
& =\left(p+q-k,\left[a \alpha^{q-k}, b \alpha^{p-k}\right], p+q-k\right) .
\end{aligned}
$$

Since the commutator subsemigroup and the derivative of any inverse semigroup $T$ are trivially closed with respect to powers of any endomorphism of $T$, then we get:

$$
(m, a, m) \in\left(\delta_{i-1}(S)\right)^{\prime} \quad \text { implies that } a \in\left(\delta_{i-2}(T)\right)^{\prime} .
$$

Let $(m, e, m)$ and $(p, f, p) \in E_{s}$, and $(m, e, m) \lambda_{\delta_{i-1}(s)}(p, f, p)$. Then, there exists a sequence:

$$
\left(n_{0}, a_{0}, n_{0}\right),\left(q_{0}, b_{0}, q_{0}\right), \ldots,\left(n_{h}, a_{h}, n_{h}\right),\left(q_{h}, b_{h}, q_{h}\right)
$$

of elements of $\delta_{i-1}(S)$ such that

$$
\begin{gathered}
(m, e, m)=\left(n_{0}, a_{0}, n_{0}\right)\left(q_{0}, b_{0}, q_{0}\right)\left(q_{0}, b_{0}^{-1}, q_{0}\right)\left(n_{0}, a_{0}^{-1}, n_{0}\right), \\
\left(q_{h}, b_{h}, q_{h}\right)\left(n_{h}, a_{h}, n_{h}\right)\left(n_{h}, a_{h}^{-1}, n_{h}\right)\left(q_{h}, b_{h}^{-1}, q_{h}\right)=(p, f, p),
\end{gathered}
$$


and, for every $j$ such that $0 \leq j \leq h$, if $u_{j}=\min \left(m_{j}, q_{j}\right)$ we get

$$
\begin{aligned}
&\left(q_{j}, b_{j}, q_{j}\right)\left(n_{j}, a_{j}, n_{j}\right)\left(n_{j}, a_{j}^{-1}, n_{j}\right)\left(q_{j}, b_{j}^{-1}, q_{j}\right) \\
&=\left(n_{j+1}, a_{j+1}, n_{j+1}\right)\left(q_{j+1}, b_{j+1}, q_{j+1}\right)\left(q_{j+1}, b_{j+1}^{-1}, q_{j+1}\right)\left(n_{j+1}, a_{j+1}^{-1}, n_{j+1}\right) \\
& n_{j}+q_{j}-u_{j}=n_{j+1}+q_{j+1}-u_{j+1}
\end{aligned}
$$

and

$$
b_{j} \alpha^{n_{j}-u_{j}} a_{j} \alpha^{q_{j}-u_{j}} a_{j}^{-1} \alpha^{q_{j}-u_{j}} b_{j}^{-1} \alpha^{n_{j}-u_{j}}=a_{j+1} \alpha^{q_{j+1}-u_{j+1}} b_{j+1} \alpha^{n_{j+1}-u_{j+1}} b_{j+1}^{-1} \alpha^{n_{j+1}-u_{j+1}} a_{j+1}^{-1} \alpha^{q_{j+1}-u_{j+1}} .
$$

Also

$$
\begin{aligned}
m & =n_{0}+q_{0}-u_{0} \\
p & =n_{h}+q_{h}-u_{h}, \\
e & =a_{0} \alpha^{q_{0}-u_{0}} b_{0} \alpha^{n_{0}-u_{0}} b_{0}^{-1} \alpha^{n_{0}-u_{0}} a_{0}^{-1} \alpha^{q_{0}-u_{0}}, \\
f & =b_{h} \alpha^{n_{h}-u_{h}} a_{h} \alpha^{q_{h}-u_{h}} a_{h}^{-1} \alpha^{q_{h}-u_{h}} b_{h}^{-1} \alpha^{n_{h}-u_{h}}
\end{aligned}
$$

At last:

$$
(m, e, n) \lambda_{\delta_{i-1}(S)}(p, f, p) \text { implies } m=p \quad \text { and } e \lambda_{\delta_{i-2}(T)} f
$$

Let

$$
(m, a, m) \in \delta_{i}(S)=\delta\left(\delta_{i-1}(S)\right)
$$

then there exists an idempotent $(m, e, m) \lambda_{\delta_{i-1}(S)}\left(m, a^{-1} a, m\right)$ such that:

$$
(m, a e, m) \in\left(\delta_{i-1}(S)\right)^{\prime} \text {. }
$$

By (3) and (4), this implies that $e \lambda_{\delta_{i-2}(T)} a^{-1} a$ and $a e \in\left(\delta_{i-2}(T)\right)^{\prime}$. Hence:

for every $i \geqslant 1, \quad(m, a, m) \in \delta_{i}(S)$ implies $a \in \delta_{i-1}(T)$.

If $T$ is solvable of class $n$, then $\delta_{n-1}(T)$ is the first derivate subsemigroup which is commutative; thus one can easily see that $\delta_{n}(S)$ must be commutative, too, and $S$ is solvable of solvability class less than or equal to $n+1$.

Now, from Theorem 3.4 of [10] we can deduce the announced result on a simple regular $\omega$-semigroup:

CoRollary 3.5. Let $S=S\left(d, G_{i}, \gamma_{i}\right)$ be a simple regular $\omega$-semigroup. Then $S$ is solvable if and only if all the groups $G_{i}$ are solvable. If $S$ is $n$-solvable, then the greatest solvability class of the groups is $n$ or $n-1$.

REMARK 3.6. When $S=S(G, \alpha)$ is a bisimple $\omega$-semigroup, then it is solvable of class $n$ if and only if $G$ is solvable of class $n$ or $n-1$. Both of these possibilities may occur. In fact consider the two following special cases of endomorphism $\alpha$ of the group $G$ :

If the endomorphism $\alpha$ is nilpotent, that is if $\alpha^{n}(G)=1$ for some $n \geq 1$, then $\left(G^{\prime}\right)_{\alpha}=G$. As $\delta(S)$ is a Clifford semigroup, we have that if $G$ is solvable of class $n-1$, then $\delta(S)$ is solvable of class $n-1$. Thus $S$ is solvable of class $n$.

If $\alpha=\mathrm{id}_{G}$, then $(G)_{\alpha}^{\prime}=G^{\prime}$. Now, if $G$ is a solvable group of class $n$, then $G^{\prime}$ is solvable of class $n-1$. Hence $\delta(S)$ is solvable of class $n-1$ and $S$ is solvable of class $n$.

REMARK 3.7. It follows from Theorem 3.11 of [10] that the maximum group homomorphic image of a solvable inverse semigroup is solvable. The converse is not true in general; actually, here we have a new family of counter-examples. 
In [7], Munn and Reilly proved that if $\alpha$ is nilpotent, then $S(G, \alpha) / \sigma$ is isomorphic to the additive group of integers. Hence, one can easily build up bisimple $\omega$-semigroups which are not solvable, if $G$ is not solvable, but where $S / \sigma$ is solvable, since it is isomorphic to a commutative group.

\section{REFERENCES}

1. J. E. Ault, Group congruences on a bisimple $\omega$-semigroup, Semigroup Forum 10 (1975), $351-366$

2. G. R. Baird, On a sublattice of the lattice of congruences on a simple regular $\omega$-semigroup, J. Austral. Math. Soc. 13 (1972), 461-471.

3. G. R. Baird, Congruences on simple regular $\omega$-semigroups, J. Austral. Math. Soc. 14 (1972), 155-167.

4. C. Bonzini and A. Cherubini, The least commutative congruence on a regular $\omega$ semigroup, Quaderno n. 21/1987, Dipartimento di Matematica dell'Universita' di Milano.

5. B. P. Kocin, The structure of inverse ideally simple $\omega$-semigroups, Vestnik Leningrad Univ. 237 (1968), 41-50.

6. W. D. Munn, Regular $\omega$-semigroups, Glasgow Math. J. 9 (1968), 46-66.

7. W. D. Munn and N. R. Reilly, Congruences on a bisimple $\omega$-semigroup, Proc. Glasgow Math. Ass., 7 (1966), 184-192.

8. M. Petrich, Congruences on simple $\omega$-semigroups, Glasgow Math. J. 20 (1979), 87-101.

9. M. Petrich, Inverse semigroups (Wiley \& Sons, 1984).

10. B. Piochi, Solvability in inverse semigroups, Semigroup Forum 34 (1987), 287-303.

11. B. Piochi, The least commutative congruence on bisimple $\omega$-semigroup, Rapp. Dip. Mat. Univ. Siena, 158 (1987), 1-14.

Dipartimento di Matematica,

UNIVERSITÀ,

Via Saldini, 50

20133 Milano.

Dipartimento di Matematica,

UNIVERSITÀ,

Via del Capitano, 15

53100 SIENA.

Dipartimento di Matematica,

Politecnico,

PiazZa L. DA VinCl, 32

20133 Milano. 